\title{
L'histoire du «patient»: aléa, moyen, ou finalité de l'histoire médicale?
}

\section{Philip Rieder}

Digby, Anne, Making a medical living. Doctors and patients in the English market for medicine, 1720-1911, Cambridge, Cambridge University Press, 1994.

Dinges, Martin (éd.), Patients in the History of Homoeopathy, Sheffield, European Association for the History of Medicine and Health Publications, 2002.

Faure, Olivier (éd.), Praticiens, Patients et Militants de l'Homéopathie (1800-1940), [Lyon], Presses Universitaires de Lyon \& Boiron S. A., 1992.

Fissell, Mary E., Patients, Power and the Poor in XVIIIth Century Bristol, Cambridge History of Medicine, Cambridge, etc., Cambridge University Press, 1991.

Pomata, Gianna, Contracting a Cure. Patients, Healers, and the Law in Early Modern Bologna, Baltimore \& London, Harvard University Press, 1998 ( $1^{\text {re }}$ éd. italienne 1994).

Porter, Roy and Dorothy Porter, In Sickness and in Health. The British Experience 1650-1850, London, Fourth Estate, 1988.

Propulsée par une mode intellectuelle engageant à construire une histoire du quotidien, des pratiques et des petites gens ou, selon l'expression consacrée, une histoire «from below», l'histoire de la médecine est habitée par un courant fort privilégiant des acteurs «modestes» du point de vue de leur apport à l'histoire positiviste de la discipline. Dans cette nébuleuse de travaux qui débusquent les praticiens «ordinaires» et inventorient des gestes «quotidiens», il faut isoler ceux dont la finalité est de combler notre ignorance sur un acteur peu connu: le patient. Le peu d'intérêt de cette figure en apparence passive, dans la perspective d'une histoire intellectuelle des savoirs va de soi. Le patient n'y trouve une place qu'en tant qu'être singulier: il est réduit à l'importance attribuée par la postérité à sa personne et seuls des patients tels que Jean-Jacques Rousseau ou Voltaire sont aujourd'hui connus. L'appel puissant lancé par Roy Porter en 1985, et les travaux menés par ce dernier avec Dorothy Porter sur ce chantier, appellent l'émergence d'une «nouvelle»

Philip Rieder, Institut universitaire romand d'histoire de la médecine et de la santé, CMU, CH-1211 Genève 4 (philip.rieder@medecine.unige.ch). 
figure historique forte et ouvrent un véritable chantier historique sur ce sujet $^{1}$.

L'objectif de cet essai est de proposer un survol critique de ce champ aujourd'hui. L'exploitation de correspondances privées et de séries d'écrits personnels a permis de mettre à jour des biographies médicales de personnes «laïques», dévoilant de véritables stratégies individuelles entre santé et maladie ${ }^{2}$. D'autres travaux, basés sur des sources issues de fonds médicaux, principalement des correspondances et des écrits médicaux, ont amené leurs auteurs à explorer de nombreux thèmes liés à l'histoire du patient: la relation thérapeutique, l'histoire du corps, l'histoire de l'écriture de sa santé, etc. ${ }^{3}$ La production est impressionnante et l'éclatement du sujet est évident. L'objectif ici n'est pas d'en explorer sérieusement le contenu, mais d'isoler une poignée d'ouvrages publiés depuis 1985, dans le sillage des Porter, dans lesquels des interprétations importantes sur la figure du patient ou sur l'évolution historique de cette figure sont proposées.

Le livre déjà ancien de Dorothy et de Roy Porter ${ }^{4}$ constitue un bon point de départ pour aborder les évolutions récentes de l'histoire du patient ne serait-ce qu'en raison du fait que les questions méthodologiques qu'il permet d'aborder sont valides encore aujourd'hui. Qui est précisément le patient? Comment organiser la chronologie de son histoire? Quel sens historique donner aux sources laissées par des patients? Voilà quelques questions qui s'imposent à la lecture de l'ouvrage et qui lui confèrent une place privilégiée ici. Les Porter concentrent leur attention sur la période allant de 1650 à 1850. Leur ouvrage est organisé en trois sections selon une logique à la fois thématique et anthropologique. La première partie du livre concerne la gestion de la santé du point de vue du malade potentiel. La santé y est décrite comme une valeur importante. Le sens et l'importance de rester en bonne santé sont débattus à travers les genres et les âges de la vie. Les mots et les concepts employés pour gérer la santé, ainsi que ceux mis en œuvre pour la conserver, sont énumérés. La deuxième partie concerne la question de l'expérience de la maladie et contient une énumération des représentations associées à différents maux. La dernière partie est consacrée à la question de la souffrance énoncée à la première personne du singulier. Ces trois parties, alimentées par un grand nombre d'extraits de sources non médicales (dont certaines fictions) et de textes de vulgarisation, permettent aux auteurs de communi-

1 Porter 1985a.

2 Quelques exemples avec des références bibliographiques: Anselment 1996; Rieder/Barras 2001.

3 Parmi d'autres: Dinges 2002b; Louis-Courvoisier 2003; Pilloud 2000; Stolberg 1996.

4 Porter/Porter 1988. 
quer une idée active de la prise en charge par le «patient» de sa propre santé. L'absence de fil conducteur clair signale pourtant les difficultés rencontrées par les auteurs dans leur tentative de donner du sens à leur matériel.

L'importance du rôle du «patient» dans ce qu'il est désormais convenu d'appeler le marché thérapeutique transpire de ce premier livre ${ }^{5}$. Le «patient» définit lui- ou elle-même son mal être, choisit son propre soignant, arrête la fin du traitement et n'accepte, parfois, d'autre appréciation sur son état que son propre jugement. Le titre de l'ouvrage In Sickness and in Health: The British Experience, 1650-1850 évoque une phénoménologie de la santé plutôt qu'une histoire du «patient». Force est de constater que pour les auteurs, le terme «patient» - absent du titre - renvoie à la relation thérapeutique et apparaît d'office inadéquat pour désigner celui ou celle qui ne recourt pas systématiquement, voire rarement, à un soignant officiel. Le «patient» est le malade, mais aussi le non-malade soucieux de sa santé: il n'est pas question de malades, par exemple, dans toute la première section de l'ouvrage. L'ambiguïté entre le terme employé (patient) et la réalité décrite (concernant aussi bien des malades et des non-malades) est connue et présente aussi bien sous l'Ancien Régime britannique qu'aujourd'hui. Ne s'agit-il pas, dès lors, comme le suggère Porter ailleurs, d'écrire une «histoire des souffrants» («sufferers' history») ${ }^{6}$ Dans tous les cas, la collusion de deux idées dans l'appel initial de Porter, celle de travailler à une histoire de la médecine du «point de vue du patient» et celle d'écrire une histoire du «souffrant»/«patient», a contribué à un manque de clarté dans la définition de l'objet même à étudier. Peu étonnamment, la façon de définir le terme de «patient» - souffrant paraît d'emblée trop restrictif - permet de diviser les écrits historiques en deux groupes, un premier ensemble où ce terme est entendu dans le sens d'un des partenaires de la relation thérapeutique (ici: patient) et le second où le même terme est entendu, plus généralement, comme «non-médecin» (ici: «patient»).

La distinction est simpliste, mais utile quand il s'agit d'aborder la question des définitions adoptées dans la littérature spécialisée. Nonobstant la mondialisation de celle-ci, le problème se décline différemment pour chaque aire culturelle. En français, par exemple, le terme (patient) est rare au XVIII ${ }^{\mathrm{e}}$ siècle et désigne, le plus souvent, celui qui souffre de la main du bourreau ou de celle du chirurgien. L'objet historique embrassé dans la littérature historique dépasse cette limite sémantique, voire encore le cadre de la relation thérapeutique. De fait, l'objet s'est étendu pour englober tout ce qui a

5 Sur le concept de marché thérapeutique, voir Cook 1986, 28-69; McCray Beier 1987, 8-50.

6 Roy Porter le relève lui-même: Porter 1985b, 181-182. 
trait à la gestion non professionnelle de la santé sous l'Ancien Régime. Les termes choisis pour désigner le «patient» répondent aux objectifs scientifiques des auteurs. Un rapide aperçu des choix opérés dans les ouvrages passés en revue ici en est une bonne illustration. Gianna Pomata, dans la traduction anglaise de son livre, jongle à bon escient entre le terme de patient, employé lorsqu'il s'agit d'un malade clairement engagé dans une relation thérapeutique, et celui de malade («sick» ou «ill») utilisé pour désigner un souffrant ou un usager du marché thérapeutique ${ }^{7}$. Les historiens anglo-saxons se contentent souvent du terme «patient», employé indifféremment avec celui de «lay» (laïc), pour désigner les non-médecins. Le terme de non-médecin est retenu dans l'ouvrage édité par Olivier Faure en français pour traduire le terme anglais «lay». Il n'en va pas de même dans l'ouvrage de Mary E. Fissell où le patient est une «personne ordinaire», alors que sous la plume d'Anne Digby, le terme est réduit à son sens étroit pour ne plus désigner que l'acteur (passif) de la relation thérapeutique ${ }^{8}$.

La démarche des Porter dans l'ouvrage de 1988 est pionnière. La principale limite, du point de vue adopté ici, est que l'information portant sur 200 ans est présentée sans articulation chronologique. Il est vrai que certaines nuances, empruntées à d'autres histoires, égrènent le récit. Un déclin de l'interprétation supranaturelle (religieuse) de la maladie à partir de la deuxième moitié du XVII ${ }^{\mathrm{e}}$ (pp. 168-169) ou encore, l'affaiblissement de la grille de lecture astrologique au XVIII ${ }^{\mathrm{e}}$ (p. 45), sont signalés. Ces évolutions ne sont pas toujours convaincantes. Que faire de la baisse, affirmée de façon péremptoire, de l'importance des interprétations humorales des malaises au XVIII ${ }^{\mathrm{e}}$ siècle (p. 45), une baisse largement contredite par des travaux sur le corps et la santé depuis lors? ${ }^{9}$ Par ailleurs, la vision synchronique de l'ouvrage consolide le modèle décrit par l'historien de la médecine Erwin Ackerknecht, selon lequel, avant le XIX ${ }^{\mathrm{e}}$ siècle, la pratique de la médecine s'organise autour du lit du malade. Elle corrobore aussi un avatar du même modèle élaboré par le sociologue N. Jewson: le «patient» est, structurellement, le patron dans ce que le même a désigné comme la cosmologie médicale, toujours avant le $\mathrm{XIX}^{\mathrm{e}}$ siècle $^{10}$. Le récit des Porter peine à aller au-delà de ces deux interprétations, et une grande partie des auteurs ultérieurs a rencontré la même difficulté.

La période historique choisie par les Porter, la deuxième moitié du XVII ${ }^{\mathrm{e}}$ et le «long» XVIII ${ }^{\mathrm{e}}$ siècle, est celle qui est la plus souvent étudiée, en raison

7 Voir, par exemple Pomata 1998, xi-xvii.

8 Voir Fissell 1991, 1; Faure 1992, 4; Digby 1994, 1-8.

9 Voir les références citées dans les notes 2, 3 et 20.

10 Ackerknecht 1982; Jewson 1976. 
notamment de l'abondance de sources. En conséquence, l'image du «patient» de la fin de l'Ancien Régime tend à être assimilée à celle du «patient traditionnel», c'est-à-dire à une image figée, projetée par défaut sur une grande partie de la période entre l'Antiquité et le XIX ${ }^{\mathrm{e}}$ siècle. Ainsi, la périodisation retenue est peu marquée et l'histoire des usagers des services médicaux avant la médecine moderne se trouve associée soit à celle de la société traditionnelle sur un modèle éthnologique ${ }^{11}$ soit à une réalité générale projetée sur la période précédant celle présentée par l'histoire classique des idées médicales comme menant à la «médecine moderne» ou «scientifique» ${ }^{12}$. Anne Digby est l'auteur qui énonce le plus clairement l'impasse. Elle constate, dès l'introduction de son ouvrage, la difficulté d'aller au-delà d'«une anthologie fascinante de récits personnels de maladie (illness)» ${ }^{13}$. Elle cherche dans son ouvrage, riche en sources produites par des «patients», à contourner l'obstacle en focalisant l'attention sur les caractéristiques financières des relations entre médecins et malades sur le long terme (1720-1911). Un des apports les plus appréciables de cet effort est de remettre clairement en question la rupture dans la relation thérapeutique, à la fin du XVIII ${ }^{\mathrm{e}}$ siècle, affirmée à la fois par Ackerknecht, Jewson et Michel Foucault, pour souligner les éléments de continuité ${ }^{14}$. Elle démontre de manière convaincante que les parcours professionnels et le succès financier des praticiens sont encore largement tributaires de l'accueil que leur réservent les patients après cette date ${ }^{15}$. De même, l'évolution de l'asymétrie propre à la relation thérapeutique, analysée socialement (par classe, genre et âge), trahit des aléas bien plus complexes que les transitions suggérées par les modèles historiques et sociologiques cités. L'ouvrage de Digby déplace l'attention du patient étudié per se, vers un autre objet, la relation thérapeutique. Ce glissement illustre une des caractéristiques de l'historiographie récente. La figure du «patient» se voit bien souvent substitué par des objets touchant le vécu ou la perspective du «patient». Les rituels autour de la mort et de la vie, les stratégies d'appropriation de savoirs médicaux, la relation thérapeutique et le rôle des soignants non-médecins sont ainsi tour à tour abordés ${ }^{16}$. Et en vérité, comme

11 Voir par exemple Fissell 1991, 16-36; ou encore Duden, Barbara, The Woman beneath the Skin (Cambridge/London $\left.{ }^{1} 1987,1991\right)$.

12 Soit avant les périodes désignées comme celles de la médecine clinique (dès le début XIX ${ }^{e}$ siècle) et de la médecine de laboratoire (dès le dernier tiers du XIX ${ }^{\mathrm{e}}$ siècle). Ackerknecht 1982; Shryock 1956.

13 Toutes les traductions ici sont les miennes.

14 Concernant les deux premiers, voir note 10. Pour les idées de Foucault, voir Foucault 1972 et 1975.

15 Particulièrement par le biais des accouchements et de la médecine des enfants: Digby 1994, 254-295.

16 Ces thèmes sont déjà énumérés par Porter au milieu des années quatre-vingt. Porter 1985a. 
Eberhard Wolff a pu le constater dans son survol de la littérature allemande, l'histoire du «patient» se trouve englobée dans de nombreux domaines voisins ${ }^{17}$. Ainsi, faire de l'histoire de la médecine du «point de vue du patient» a souvent pour résultat de laisser de côté la figure du «patient».

C'est notamment le cas dans le très stimulant ouvrage de Mary E. Fissel. Fissel concentre son analyse sur le XVIII ${ }^{\mathrm{e}}$ siècle. Son objectif affirmé est de réévaluer l'évolution des institutions caritatives et médicales à Bristol, à cette époque, en optant consciemment pour le point de vue des «patients» (Fissel 1991). Les sources employées pour décrire la situation d'avant la mise en place d'hôpitaux modernes, dans le sens d'espaces spécialisés dans les soins, embrassent un éventail large. Elles sont essentiellement non médicales et communiquent une image arrêtée d'une médecine traditionnelle, caractérisée avant tout comme étant un monde où tous partagent les mêmes notions relatives au corps et à la santé. Sa volonté d'aborder le cas des pauvres l'engage à chercher à reconstituer leurs stratégies d'utilisation des institutions caritatives et hospitalières, une démarche qui lui permet de décrire comment les «patients» parviennent à utiliser ces institutions en fonction de leurs propres besoins au début du XVIII ${ }^{\mathrm{e}}$ siècle. Ainsi, si les fondateurs de l'hôpital de Bristol projettent d'y aider les familles locales, l'hôpital se transforme rapidement en substitut familial en accueillant de jeunes adultes déracinés (p. 105). Fissel propose ici l'ébauche d'une approche centrée sur l'angle de la «demande» du marché thérapeutique. Malheureusement, cette préoccupation n'est pas poursuivie dans la suite de son ouvrage où, en évoquant la création de nouveaux espaces de soins (le dispensaire et l'hôpital) à partir d'archives professionnelles, elle change de perspective. Ses développements s'avèrent intéressants quant aux stratégies professionnelles des soignants, mais, du point de vue du «patient», ils confortent simplement les modèles susmentionnés de Jewson, Ackerknecht, et surtout de Foucault. La volonté de cerner la perspective du «patient» démuni dans les derniers chapitres de son livre l'amène à minimiser la continuité des attitudes anciennes, voire la capacité du «patient» à résister au modèle médical: sa démonstration s'appuie sur le changement d'attitude, bien documenté, des soignants.

Les chapitres de Fissell sont admirablement bien documentés et constituent autant de contributions fortes à l'histoire de la santé, à celle de la clinique, des métiers de la santé, voire encore de la charité. Quant à son interprétation globale de l'histoire du «patient», force est de constater qu'elle ne convainc pas. Fissell déduit de sources médicales (institutionnelles et professionnelles) l'objectivation du «patient» et son aliénation de sa propre

17 Wolff 1998, 209. 
santé à partir de la fin du XVIII ${ }^{\mathrm{e}}$ siècle et du début du siècle suivant. Le choix d'articuler son interprétation en fonction de l'évolution du savoir et des institutions médicales plutôt que sur la continuité des attitudes non médicales, comme c'est le cas chez Digby, induit une perspective partielle de l'histoire du «patient». Schématiquement, le «patient» défini comme une «personne ordinaire» au début du livre devient imperceptiblement un «pauvre» à la fin de celui-ci. L'impression gagnée à la lecture de son ouvrage demeure que son modèle explicatif conditionne à la fois les sources qu'elle met à contribution et son interprétation. La volonté du soignant de trouver une maladie particulière, objective, est-elle vraiment confinée à la sphère médicale? Le patient n'en est-il pas également le véhicule? De même, si certains non-médecins s'opposent à des autopsies au XIX ${ }^{\mathrm{e}}$ siècle, la commande d'autopsies privées par des particuliers au siècle précédant (Rousseau, par exemple, réclame la sienne en 1763), n'indique-t-elle pas l'intérêt de «patients» pour des entités nosologiques plus précises? La volonté de comprendre ce qui se passe sous la peau, propre à certains médecins à la fin de l'Ancien Régime, n'est-elle pas partagée par des non-médecins?

L'ouvrage de Gianna Pomata consacré aux différends entre malades et praticiens à Bologne échappe à de telles difficultés méthodologiques (Pomata, 1998). L'auteur reconstruit le monde des valeurs propre aux échanges entre patients et soignants (entendus dans un sens large) dans la ville de Bologne du XVI ${ }^{\mathrm{e}}$ siècle au XVIII ${ }^{\mathrm{e}}$ et démontre l'importance de la notion de promesse de soins sur le marché médical à cette époque: le soignant s'engage par avance à guérir un malade contre une somme arrêtée et en un temps convenu. Ces «pactes de guérison» sont parfois oraux, mais peuvent également être conclus devant un notaire. La pratique dépasse la seule sphère locale et est attestée dans d'autres contrées européennes. Pomata analyse au quotidien les aléas de ce marché médical au XVI ${ }^{\mathrm{e}}$ siècle à travers le tribunal des protomedicati et retrace la diminution progressive de la pratique de promesse jusqu'à la voir disparaître du marché des soins au XVIII ${ }^{\mathrm{e}}$ siècle. L'histoire de la promesse de guérison permet d'intégrer une articulation dans l'histoire de la figure du patient: au début de l'époque moderne, celui-ci négocie ouvertement avec des soignants et ne paye les soins, finalement, que s'il en est satisfait. En dépit d'une certaine continuité de la promesse de guérir, au cours de l'époque moderne le patient est progressivement amené à payer chaque conseil et chaque service médical ${ }^{18}$.

18 La continuité des pratiques anciennes est attestée à différents endroits. Certains soins, par exemple, sont assurés selon ce principe dans le cadre de la «Poor law» en Angleterre encore au XVIII' siècle: Digby 1994, 228. 
Les deux travaux collectifs sur l'histoire de l'homéopathie apportent un autre éclairage sur l'histoire du «patient» envisagé sur le long terme (Faure 1992; Dinges 2002a). L'essor et l'épanouissement de cette école médicale particulière sont largement dus à la demande des patients eux-mêmes. Le malade est apparemment patient. Cependant, en l'absence d'un nombre suffisant de thérapeutes homéopathes, armé d'ouvrages et publications de vulgarisation, le «patient» prend souvent lui-même en charge sa propre cure, voire celle de ses proches et de ses amis ${ }^{19}$. Dans certains contextes, des nonmédecins (ou des soignants sans doctorat) sont amenés plus ou moins légalement à pratiquer. Le «patient» est ainsi plus qu'un simple malade et doit être entendu dans un sens large. Le malade recourrant à l'homéopathie, écrit Michael Stolberg, échappe en partie à l'objectivation physique en cours dans l'histoire allopathique et offre ainsi des exemples de continuité des gestes et stratégies subjectives repérables au cours de l'Ancien Régime ${ }^{20}$. La continuité de principes humoraux, attestée dans les lettres de patients adressées à des homéopathes, est flagrante au XIX ${ }^{\mathrm{e}}$ siècle alors que l'impact des événements marquants de l'histoire classique des idées médicales (anesthésie, antisepsie, etc.) sur le contenu des mêmes correspondances s'avère faible ${ }^{21}$. L'histoire de l'homéopathie telle qu'elle se décline dans ces deux ouvrages signale l'autonomie relative du «patient» jusqu'au $\mathrm{XX}^{\mathrm{e}}$ siècle ${ }^{22}$, certains malades consultant simultanément ou alternativement homéopathes et allopathes $^{23}$. Le développement d'associations de patients, évoqués par plusieurs auteurs, mais abordé sérieusement par Eberhard Wolff, atteste de la continuité d'une volonté de conserver le libre arbitre du non-médecin, de diffuser les pratiques homéopathiques et, dans une certaine mesure, de contrôler la qualité des soins ${ }^{24}$. A la suite d'Olivier Faure, on peut conclure que l'histoire sociale de l'homéopathie illustre le fait qu'au cours des $\mathrm{XIX}^{\mathrm{e}}$ et $\mathrm{XX}^{\mathrm{e}}$ siècles,

19 Ce rôle est reconnu. Voir l'article de Werner Friedrich Kümmer, «L'état de la recherche en histoire de l'homéopathie», dans: Faure 1992, 27-28. De nombreux soignants homéopathes sont autodidactes ou «non-médecins». Voir par exemple Brade, Anne-Elisabeth, "Sören Jensen: a Danish Homoeopath, his Time and his Patients", and Gijwijt-Hofstra, Marijke, "The Haverhoeks and their Patients: the Popularity of Unqualified Homoeopaths in the Netherlands in the Early Twentieth Century", dans: Dinges 2002a, 186 et 213-231.

20 Stolberg, Michael, “The Experience of Illness and the Doctor-Patient Relationship in Samuel Hahnemann's Patient Correspondence", dans: Dinges 2002a, 67.

21 Dinges 2002a, 69-70.

22 Faure, Olivier, «La clientèle d'un homéopathe parisien au XX ${ }^{\mathrm{e}}$ siècle», dans: Faure 1992 , 184-185.

23 Par exemple Brade, op. cit. (supra note 19), 191-192; Svana-Pétursdóttir, Sigrídur, “'Patients don't care from where the cure comes': Patients' Choice of a Homoeopathic Folk Healer in Iceland", dans: Dinges 2002a, 178-180; Nachtmann, Walter, «Les malades face à Hahnemann (d'après leur correspondance juin-octobre 1832)», dans: Faure 1992, 151-152.

24 Wolff, Eberhard, «Le rôle du mouvement des non-médecins dans le développement de l'homéopathe en Allemagne», dans: Faure 1992, 197-230. 
les problèmes de santé ne sont pas du seul ressort du spécialiste. Des nonmédecins ont également une «sensibilité autonome à la santé»" 25.

Par ailleurs, la campagne de Hahnemann contre les pharmaciens est orchestrée au nom de la notion de contrat de guérison ${ }^{26} \ldots$ Ce phénomène, répété dans différentes contributions, suggère, comme l'amène à penser l'étude d'Anne Digby sur les relations financières entre malades et soignants, que la pratique médicale peine à s'affranchir des valeurs de la promesse de guérison (Digby 1994, 36). Du moins est-il évident que le «patient» n'honore pas facilement la note que lui adresse le soignant. Contrairement aux remèdes achetés en boutique, le conseil médical n'est pas toujours associé à un service ou à une commodité payée. L'idée de rémunérer un service médical en tant que tel, idée qui s'impose progressivement dans le cadre professionnel, comme le démontre Pomata, rencontre une résistance importante auprès des usagers. Il y a de quoi voir là une autre réfutation du principe de la soumission du patient à la médecine allopathique aux XIX ${ }^{\mathrm{e}}$ et $\mathrm{XX}^{\mathrm{e}}$ siècles inhérent aux modèles de Jewson et de Foucault. L'histoire particulière de l'homéopathie, portant sur plus d'un siècle de pratiques, va dans le même sens et signale la dynamique propre à la demande sur le marché thérapeutique.

Les difficultés méthodologiques et les questionnements sans réponse demeurent encore nombreuses dans le chantier sur le «patient» tel qu'il se profile aujourd'hui. Les questions de chronologie, de gestion qualitative de données trouvées dans des proportions plutôt quantitatives et le rapport, toujours difficile à aborder, entre le parcours individuel et une réalité plus générale, demeurent. Une question fondamentale est centrale dans l'ensemble de cette littérature: le patient est restitué dans un cadre médical que l'on peut considérer, selon les standards d'aujourd'hui, comme étant peu efficace. De là à intégrer cette considération dans l'interprétation historique, il n'y a qu'un pas. Porter et Porter, par exemple, sont largement pessimistes face à l'efficacité ancienne, même si la question n'est pas abordée clairement. Les appréciations sont lapidaires. En commentant, par exemple, la bonne santé en tant qu'idéal, ils écrivent: «Les maux étaient insupportables, la médecine dégouttante et généralement sans effet de toute façon» (Porter et Porter, p. 26). Digby, tout en reconnaissant intelligemment la difficulté d'évaluer l'efficacité historique de pratiques médicales sur une population donnée (Digby 1994, 75-77), évoque, sans les expliciter, les «limites du savoir médical» (p. 214), réduit l'efficacité thérapeutique des eaux minérales à «l'effet

25 Faure, Olivier, «Conclusion», dans: Faure 1992, 233.

26 Michalak, Michael, «Hahnemann et les pharmaciens», dans: Faure 1992, 114. 
placebo» (p. 211) et base une grande partie de son argumentation, sans offrir de commentaire critique, sur l'inefficacité de la médecine ancienne: l'incertitude quant à l'effet des remèdes serait responsable, notamment, du développement de thérapies douces dans lesquelles elle inclut les régimes (p. 201) ${ }^{27}$.

De tels commentaires et interprétations sont fréquents dans les travaux historiques abordant la question du monde des soins d'Ancien Régime. Ils donnent à voir une réaction de «patient» d'aujourd'hui face à l'évocation de thérapies anciennes. Ces prises de position renvoient au sens commun de ce qu'est une «bonne» ou une «mauvaise» médecine et sous-entendent ce qui est efficace en matière de thérapeutique du point de vue d'un commentateur clairement enraciné dans le monde contemporain ${ }^{28}$. Or, la lecture de correspondances et d'écrits personnels rédigés sous l'Ancien Régime convainc du fait que l'efficacité est conçue dans une perspective relative et individuelle. Ainsi, les commentaires sur «l'inefficacité» ancienne s'avèrent peu utiles pour comprendre le monde médical du passé. Afin de rendre compte historiquement de la santé au quotidien, il est essentiel de se départir de la grille de lecture suggérée par nos connaissances médicales (aussi faibles soientelles). Certains historiens vont assez loin. Barbara Duden, par exemple, n'hésite pas à affirmer être prête à croire qu'au XVIII ${ }^{\mathrm{e}}$ siècle il était possible d'uriner par la bouche ou de voir les menstrues s'écouler par différentes plaies $^{29}$. Sans aller aussi loin, l'anthropologie médicale suggère une approche phénoménologique qui permet d'appréhender la logique propre aux actes ainsi que les représentations liées à la santé et à la maladie. C'est ainsi qu'il est possible de décrire la voie explorée par Pomata, Fissell et par la plupart des auteurs travaillant sur l'histoire de l'homéopathie. Fissell constate, par exemple, l'importance de l'enracinement de la logique de la médecine humorale - aussi bien pour les soignants que pour les soignés (Fissell 1991, 16-36). En conséquence, elle nuance la notion de guérison trouvée dans les archives hospitalières pour en faire un concept proche de l'idée de retour à un équilibre initial plutôt que dans le sens de la réparation d'une partie ou d'une fonction corporelle (pp. 107-108) ${ }^{30}$.

27 Outrepasser la notion «d'efficacité médicale» dans le sens contemporain du concept est un des moteurs du regain d'intérêt actuel pour l'histoire de la thérapeutique. Voir par exemple: Faure 1999; Maehle 1999.

28 L'évolution de l'efficacité thérapeutique est ainsi souvent dépeint du point de vue de la biomédecine: Digby 1994, 97.

29 Duden 1991, 6-7.

30 Barbara Duden a démontré que le concept de «mieux être» ou de guérison est historiquement contingent: Duden 1991, 156 et 172. 
Ainsi, en guise de conclusion, le courant historique dont les ouvrages passés en revue ici sont issus s'avère riche et ouvre de nombreuses perspectives. La figure du «patient» s'y construit le plus souvent par opposition à d'autres objets, voire d'autres domaines historiques. L'utilité heuristique de ces travaux pour l'histoire de la médecine est importante. L'histoire du «patient» qui en émerge incite à nuancer l'importance socioculturelle des ruptures épistémologiques attestées dans le récit classique de l'histoire des savoirs médicaux, voire à signaler des modalités de diffusion et d'appropriations de savoirs savants et populaires. Il permet, notamment par l'étude de pratiques alternatives comme celles de l'homéopathie, d'attester de la capacité de résistance face à l'idéologie médicale dominante. Cette histoire donne un aperçu de la réalité du marché médical, des pratiques de soignants et de l'autonomie interprétative et thérapeutique des malades à chaque période envisagée. Ce sont les apports les plus rentables du point de vue historiographique, car finalement, c'est en regard de l'ensemble de l'historiographie de la médecine que l'objet se construit. La perspective porte préjudice, par contre, à une construction holistique de la figure du patient lequel se trouve noyée dans d'autres histoires (celles des praticiens, du corps, de la relation thérapeutique, etc.). La littérature passée en revue ici atteste de la difficulté d'isoler cette figure pourtant omniprésente. Ainsi, la question de savoir s'il est possible ou même utile d'envisager le «patient» en tant que figure historique, demeure.

Aujourd'hui encore, il est tentant de terminer ce survol en avançant l'intérêt d'une histoire diachronique du patient pour celle, plus traditionnelle, de la médecine. Ce n'est pourtant qu'en sortant cette histoire du carcan d'une histoire utile à d'autres fins qu'il sera possible d'en faire un objet historique à part entière. Car, au-delà de son utilité pour d'autres chantiers, le nonmédecin est également le véhicule, voire la voix, de cultures de la santé originales. La figure du «patient» incarne une culture médicale laïque et à ce titre mérite une attention soutenue. Les ouvrages passés en revue ici contribuent à cerner ces cultures médicales, et l'amélioration de telles connaissances doit être sur l'agenda de la recherche dans ce domaine. L'histoire du «patient» a une raison d'être en apportant des données sur les attitudes face à la santé, à la maladie, voire sur la demande régissant le marché thérapeutique. Le soignant lui-même n'est-il pas un «patient» dans le sens large du terme tel qu'il a été défini plus haut? Et si le «patient» est aujourd'hui utile à la connaissance historique, c'est avant tout en apportant une meilleure compréhension des acteurs historiques, de leur propre façon d'appréhender leur environnement et de se comprendre eux-mêmes. 


\section{Bibliographie}

Ackerknecht, Erwin H., A Short History of Medicine (Baltimore/London 1982 [1968])

Anselment, Raymond A., “'The Want of Health': An Early Eighteenth-Century Self-Portrait of Sickness", Literature and Medicine 15 (1996) 225-243

Cook, Harold J., The Decline of the Old Medical Regime in Stuart London (Ithaca/London 1986)

Digby, Anne, Making a Medical Living. Doctors and patients in the English market for medicine, 1720-1911 (Cambridge 1994)

Dinges, Martin (éd.), Patients in the History of Homoeopathy (Sheffield 2002a)

- «Männlichkeitskonstruktion im medizinischen Diskurs um 1830: Der Körper eines Patienten von Samuel Hahnemann», dans: Jürgen Martschukat (éd.), Geschichte schreiben mit Foucault (Frankfurt/New York 2002b) 99-125

Duden, Barbara, The Woman beneath the Skin (Cambridge/London $\left.{ }^{1} 1987,1991\right)$

Faure, Olivier (éd.), Praticiens, Patients et Militants de l'Homéopathie (1800-1940) (Lyon 1992)

- Les thérapeutiques: savoirs et usages (Oullins 1999)

Fissell, Mary E., Patients, Power and the Poor in XVIIIth Century Bristol (Cambridge 1991)

Foucault, Michel, Naissance de la clinique (Paris 1972)

- Surveiller et punir (Paris 1975)

Jewson, N. D., "The Disappearance of the Sick-man from Medical Cosmology, 1770-1870", Sociology 10 (1976) 225-245

Louis-Courvoisier, Micheline, «Qu'est-ce qu'un malade sans son corps? L'objectivation du corps vue à travers les lettres de consultations adressées au Dr Tissot (1728-1797)», dans: Annette Kreis-Schinck, Franziska Frei Gerlach, Claudia Opitz, Béatrice Ziegler (éds), Concepts du corps. Contribution aux études genres interdisciplinaires (Münster 2003) 299-310

Maehle, Andreas-Holger (éd.), Drugs on Trial: Experimental Pharmacology and Therapeutic Innovation in the Eighteenth Century (Amsterdam 1999)

McCray Beier, Lucinda, Sufferers and Healers (London/New York 1987)

Pilloud, Séverine, «Mettre les maux en mots, médiations dans la consultation épistolaire au XVIII ${ }^{\mathrm{e}}$ siècle: les malades du Dr Tissot», Bulletin Canadien de l'histoire de la médecine 16 (2000) 215-245

Pomata, Gianna, Contracting a cure. Patients, Healers, and the Law in Early Modern Bologna (Baltimore 1998, $1^{\text {re }}$ éd. italienne 1994)

Porter, Roy, “Introduction”, dans: Roy Porter (éd.), Patients and Practitioners (Cambridge 1985a) $1-22$

- "The Patient's View. Doing Medical History from Below", Theory and Society 4 (1985b) 175-198

Porter, Roy/Dorothy Porter, In Sickness and in Health. The British Experience 1650-1850 (London 1988)

Rieder, Philip/Vincent Barras, «Santé et maladie chez Saussure», dans: René Sigrist (éd.), H.-B. de Saussure (Genève 2001) 501-524

Shryock, Richard Harrison, Histoire de la médecine moderne: facteur scientifique, facteur social (Paris 1956)

Stolberg, Michael, «Mein äskulapisches Orakel!〉 Patientenbriefe als Quelle einer Kulturgeschichte der Krankheitserfahrung im 18. Jahrhundert», Österreichische Zeitung für Geschichte 7 (1996) 385-404

Wolff, Eberhard, "Perspectives on Patients' History: Methodological Considerations on the Example of Recent German-speaking Literature”, Bulletin Canadien d'Histoire de la Médecine 15 (1998) 207-228 